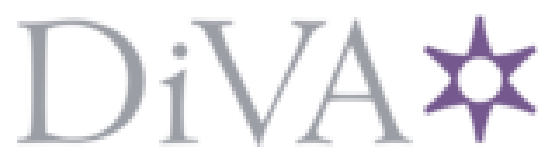

http://www.diva-portal.org

This is the published version of a paper published in New England Journal of Medicine.

Citation for the original published paper (version of record):

Chinot, O., Wick, W., Mason, W., Henriksson, R., Saran, F. et al. (2014)

Bevacizumab plus radiotherapy-temozolomide for newly diagnosed glioblastoma.

New England Journal of Medicine, 370(8): 709-722

http://dx.doi.org/10.1056/NEJMoa1308345

Access to the published version may require subscription.

N.B. When citing this work, cite the original published paper.

Permanent link to this version:

http://urn.kb.se/resolve?urn=urn:nbn:se:umu:diva-89503 


\section{Bevacizumab plus Radiotherapy-Temozolomide for Newly Diagnosed Glioblastoma}

\author{
Olivier L. Chinot, M.D., Wolfgang Wick, M.D., Warren Mason, M.D., \\ Roger Henriksson, M.D., Frank Saran, M.D., Ryo Nishikawa, M.D., \\ Antoine F. Carpentier, M.D., Ph.D., Khe Hoang-Xuan, M.D., Ph.D., \\ Petr Kavan, M.D., Ph.D., Dana Cernea, Ph.D., Alba A. Brandes, M.D., \\ Magalie Hilton, M.Sc., Lauren Abrey, M.D., and Timothy Cloughesy, M.D.
}

\section{A BSTRACT}

\section{BACKGROUND}

Standard therapy for newly diagnosed glioblastoma is radiotherapy plus temozolomide. In this phase 3 study, we evaluated the effect of the addition of bevacizumab to radiotherapy-temozolomide for the treatment of newly diagnosed glioblastoma.

\section{METHODS}

We randomly assigned patients with supratentorial glioblastoma to receive intravenous bevacizumab (10 mg per kilogram of body weight every 2 weeks) or placebo, plus radiotherapy (2 Gy 5 days a week; maximum, 60 Gy) and oral temozolomide (75 mg per square meter of body-surface area per day) for 6 weeks. After a 28-day treatment break, maintenance bevacizumab $(10 \mathrm{mg}$ per kilogram intravenously every 2 weeks) or placebo, plus temozolomide (150 to $200 \mathrm{mg}$ per square meter per day for 5 days), was continued for six 4-week cycles, followed by bevacizumab monotherapy (15 mg per kilogram intravenously every 3 weeks) or placebo until the disease progressed or unacceptable toxic effects developed. The coprimary end points were investigator-assessed progression-free survival and overall survival.

\section{RESULTS}

A total of 458 patients were assigned to the bevacizumab group, and 463 patients to the placebo group. The median progression-free survival was longer in the bevacizumab group than in the placebo group (10.6 months vs. 6.2 months; stratified hazard ratio for progression or death, $0.64 ; 95 \%$ confidence interval [CI], 0.55 to 0.74 ; $\mathrm{P}<0.001)$. The benefit with respect to progression-free survival was observed across subgroups. Overall survival did not differ significantly between groups (stratified hazard ratio for death, $0.88 ; 95 \% \mathrm{CI}, 0.76$ to $1.02 ; \mathrm{P}=0.10$ ). The respective overall survival rates with bevacizumab and placebo were $72.4 \%$ and $66.3 \%$ at 1 year $(\mathrm{P}=0.049)$ and $33.9 \%$ and $30.1 \%$ at 2 years $(\mathrm{P}=0.24)$. Baseline health-related quality of life and performance status were maintained longer in the bevacizumab group, and the glucocorticoid requirement was lower. More patients in the bevacizumab group than in the placebo group had grade 3 or higher adverse events (66.8\% vs. $51.3 \%)$ and grade 3 or higher adverse events often associated with bevacizumab (32.5\% vs. $15.8 \%$ ).

\section{CONCLUSIONS}

The addition of bevacizumab to radiotherapy-temozolomide did not improve survival in patients with glioblastoma. Improved progression-free survival and maintenance of baseline quality of life and performance status were observed with bevacizumab; however, the rate of adverse events was higher with bevacizumab than with placebo. (Funded by F. Hoffmann-La Roche; ClinicalTrials.gov number, NCT00943826.)
From Aix-Marseille University, Assistance Publique-Hôpitaux de Marseille, Service de Neuro-Oncologie, Centre Hospitaliere Universitaire Timone, Marseille (O.L.C.), UFR de Santé, Médecine et Biologie Humaine, Bobigny (A.F.C.), and Assistance Publique-Hôpitaux de Paris (AP-HP), Hôpital Avicenne, Service de Neurologie, Université Paris 13 (A.F.C.), and AP-HP, Université Pierreet-Marie-Curie, Group Hospitalier PitiéSalpêtrière (K.H.-X.), Paris - all in France; University Hospital of Heidelberg, Department of Neurooncology, and German Cancer Consortium, German Cancer Research Center, Heidelberg, Germany (W.W.); Princess Margaret Hospital, Toronto (W.M.), and McGill University, Montreal (P.K.) - both in Canada; Regional Cancer Center, Stockholm Gotland, Karolinska, Stockholm, and the Department of Radiation Sciences and Oncology, Umeå University, Umeå - both in Sweden (R.H.); the Royal Marsden National Health Service Foundation Trust, Sutton, Surrey, United Kingdom (F.S.); Saitama Medical University, Saitama, Japan (R.N.); Oncology Institute "Ion Chiricuta," Cluj-Napoca, Romania (D.C.); Medical Oncology Department, Azienda Unità Sanitaria Locale, Bologna, Italy (A.A.B.); F. Hoffmann-La Roche, Basel, Switzerland (M.H., L.A.); and University of California, Los Angeles, Los Angeles (T.C.). Address reprint requests to Dr. Chinot at Aix-Marseille University, AP-HM, Service de Neuro-Oncologie, CHU Timone, 264, Rue Saint Pierre, 13005 Marseille, France, or at olivier.chinot@ap-hm.fr.

N Engl J Med 2014;370:709-22. DOI: 10.1056/NEJMoa1308345 Copyright (๑) 2014 Massachusetts Medical Society. 
T

UMOR PROGRESSION IN GLIOBLASTOMA, the most common primary brain cancer, ${ }^{1,2}$ is associated with deterioration in neurocognitive function, ${ }^{3,4}$ decreased functional independence, ${ }^{5}$ and a progressive decrease in health-related quality of life. ${ }^{6,7}$ After surgical resection, the standard of care for patients with newly diagnosed glioblastoma and a good Karnofsky performance score $(\geq 70$, on a scale of 0 to 100 , with higher numbers indicating better functioning) is concurrent radiotherapy and temozolomide, followed by adjuvant temozolomide. ${ }^{8-11}$ The prognosis remains poor; no further improvements in outcomes have been documented since the introduction of radiotherapy-temozolomide therapy in 2005.

Glioblastomas are characterized by overexpression of vascular endothelial growth factor A (VEGF-A), a key regulator of tumor-associated angiogenesis, ${ }^{12-15}$ and these tumors are highly vascularized. ${ }^{16}$ The results of phase $1 / 2$ studies support a role for the anti-VEGF-A molecule bevacizumab in recurrent and newly diagnosed glioblastoma. ${ }^{17-22}$ We report the results of a phase 3 trial of bevacizumab plus radiotherapy-temozolomide as compared with placebo plus radiotherapytemozolomide in patients with newly diagnosed glioblastoma.

\section{METHODS}

\section{STUDY OVERSIGHT}

The Avastin in Glioblastoma (AVAglio) study (BO21990) was a randomized, double-blind, placebo-controlled trial sponsored by F. HoffmannLa Roche and designed by the AVAglio steering committee (see the Supplementary Appendix, available with the full text of this article at NEJM .org) and the sponsor. We conducted the study at 120 sites in 23 countries. The steering committee provided oversight of the overall scientific integrity of the study. The protocol (available at NEJM.org) was approved by the applicable independent ethics committees and institutional review boards. Real-time monitoring of safety events was overseen by an independent data and safety monitoring board. The study adhered to the principles of the Declaration of Helsinki and the Guidelines for Good Clinical Practice. All the authors signed confidentiality agreements with the sponsor regarding the data. The data were collected by the sponsor and were analyzed by an author employed by the sponsor, who vouches for the accuracy of the data. Medical writing assistance was provided by Gardiner-Caldwell Communications and paid for by the sponsor. All the authors vouch for the adherence of the study to the protocol and made the decision to submit the manuscript for publication.

\section{PATIENTS}

Patients 18 years of age or older with newly diagnosed, histologically confirmed, supratentorial glioblastoma were eligible for participation in the study. Additional inclusion criteria were a World Health Organization (WHO) performance status of 2 or lower (on a scale of 0 to 5 , with higher numbers indicating decreasing performance); the use of stable or decreasing glucocorticoid doses within the 5 days before randomization; adequate healing of craniotomy or cranial-biopsy site; adequate hematologic, hepatic, and renal function; and acceptable blood coagulation levels. Investigators submitted available tumor tissue blocks for pathological central review and analysis of status with respect to O-6-methylguanineDNA methyltransferase (MGMT). Treatment had to be initiated between 29 and 48 days after the most recent surgery. Patients were excluded if they had evidence of recent symptomatic intracranial hemorrhage on magnetic resonance imaging (MRI), prior chemotherapy or immunotherapy for glioblastoma or low-grade astrocytoma, prior radiotherapy to the brain, a history of intracranial abscess within 6 months before randomization, or a serious nonhealing wound. All patients were required to give written informed consent before enrollment.

\section{RANDOMIZATION AND TREATMENT}

Patients were randomly assigned, in a 1:1 ratio, to bevacizumab or placebo. Randomization was performed centrally with the use of an interactive voice-response system, with stratification according to study region (Western Europe, Eastern Europe, Asia, United States, or other) and recursive partitioning analysis class (III, IV, or V). ${ }^{23}$ (There are six recursive partitioning analysis classes, of which classes III, IV, V, and VI are used to categorize glioblastoma, with higher numbers representing a worse prognosis. Class VI patients were considered too frail to participate in this study.) 
The study sponsor, study investigators, and patients were unaware of the study-group assignments. Unblinding of the assignments was allowed at any time for safety reasons or at the time of disease progression if deemed necessary by the investigator.

After undergoing surgical resection or biopsy, patients received concurrent radiotherapy (60 Gy administered as 2-Gy fractions 5 days per week) and oral temozolomide ( $75 \mathrm{mg}$ per square meter of body-surface area per day for a maximum of 49 days), in combination with intravenous bevacizumab (10 mg per kilogram of body weight) or placebo every 2 weeks. The last concurrent doses of temozolomide and bevacizumab or placebo were administered on the day of the last dose of radiotherapy. The concurrent-therapy phase was followed by a 28 -day treatment break.

In the maintenance phase, patients received temozolomide ( $150 \mathrm{mg}$ per square meter per day on days 1 to 5 during the first cycle and $200 \mathrm{mg}$ per square meter per day during subsequent cycles if unacceptable toxic effects did not devel$\mathrm{op}^{24}$ ) plus intravenous bevacizumab (10 $\mathrm{mg}$ per kilogram) or placebo every 2 weeks, for six 4-week cycles. In the monotherapy phase, intravenous bevacizumab (15 $\mathrm{mg}$ per kilogram) or placebo was continued every 3 weeks until the disease progressed or unacceptable toxic effects developed.

\section{ASSESSMENTS}

The determination of progression was based on imaging assessment (MRI), clinical assessment, and glucocorticoid use ${ }^{25}$ (Table S1 in the Supplementary Appendix). Radiographic criteria were adapted to address specific concerns related to the effect of antiangiogenic therapy on imaging. Specifically, assessment of nonenhancing tumor components was included, and a specific algorithm was used to assess pseudoprogression. ${ }^{25}$ These adaptations are consistent with current international consensus guidelines. ${ }^{26}$ Assessments were carried out at baseline; 28 days after completion of the concurrent-therapy phase; during cycles 2, 4, and 6 of the maintenance phase; every 9 weeks throughout the monotherapy phase; and at the time of disease progression. Pseudoprogression was assessed at the end of the treatment break with the use of a strict algorithm, ${ }^{26}$ and confirmatory imaging was performed after two cycles of maintenance therapy.
In addition to investigator-assessed progression, radiologists at an independent review facility analyzed all MRI scans. The independent reviewers were unaware of the study-group assignments, with read-only access to previous reviews until the final imaging data set was reviewed; at completion of the study, a review of the entire scan series verified the time of progression on MRI. In a final independent review, the determination of progression was calculated with the use of a prespecified algorithm that combined the assessment of the scans by the independent reviewer with the investigator's neurologic evaluation and assessment of glucocorticoid use.

Quality of life was measured with the use of the validated core quality-of-life questionnaire (QLQ-C30) and a quality-of-life questionnaire specifically for patients with brain tumors (BN20) of the European Organization for Research and Treatment of Cancer. ${ }^{27-29}$ Patients completed the questionnaires without assistance. Five scales were prespecified for the primary analysis of deterioration-free survival: global health status, physical functioning, social functioning, motor dysfunction, and communication deficit. An additional 21 nonprespecified scales were assessed in exploratory analyses. The score on the Mini-Mental State Examination (MMSE, on which scores range from 0 to 30 , with higher scores indicating better cognitive function) was used to assess neurocognitive function (see Section 4 in the Supplementary Appendix). These assessments were performed at each disease-assessment time point (before the clinical evaluation). The Karnofsky performance status was graded by the treating physician. Adverse events were assessed throughout the study, according to National Cancer Institute Common Terminology Criteria, version 3.0. ${ }^{30}$

\section{STATISTICAL ANALYSIS}

The coprimary end points were investigatorassessed progression-free survival and overall survival. The overall 0.05 level of significance was split asymmetrically between the two coprimary end points, with 0.01 allocated to progressionfree survival and 0.04 to overall survival. For the analysis of progression-free survival, assuming median durations of 9.1 months in the group receiving bevacizumab plus radiotherapy-temozolomide 


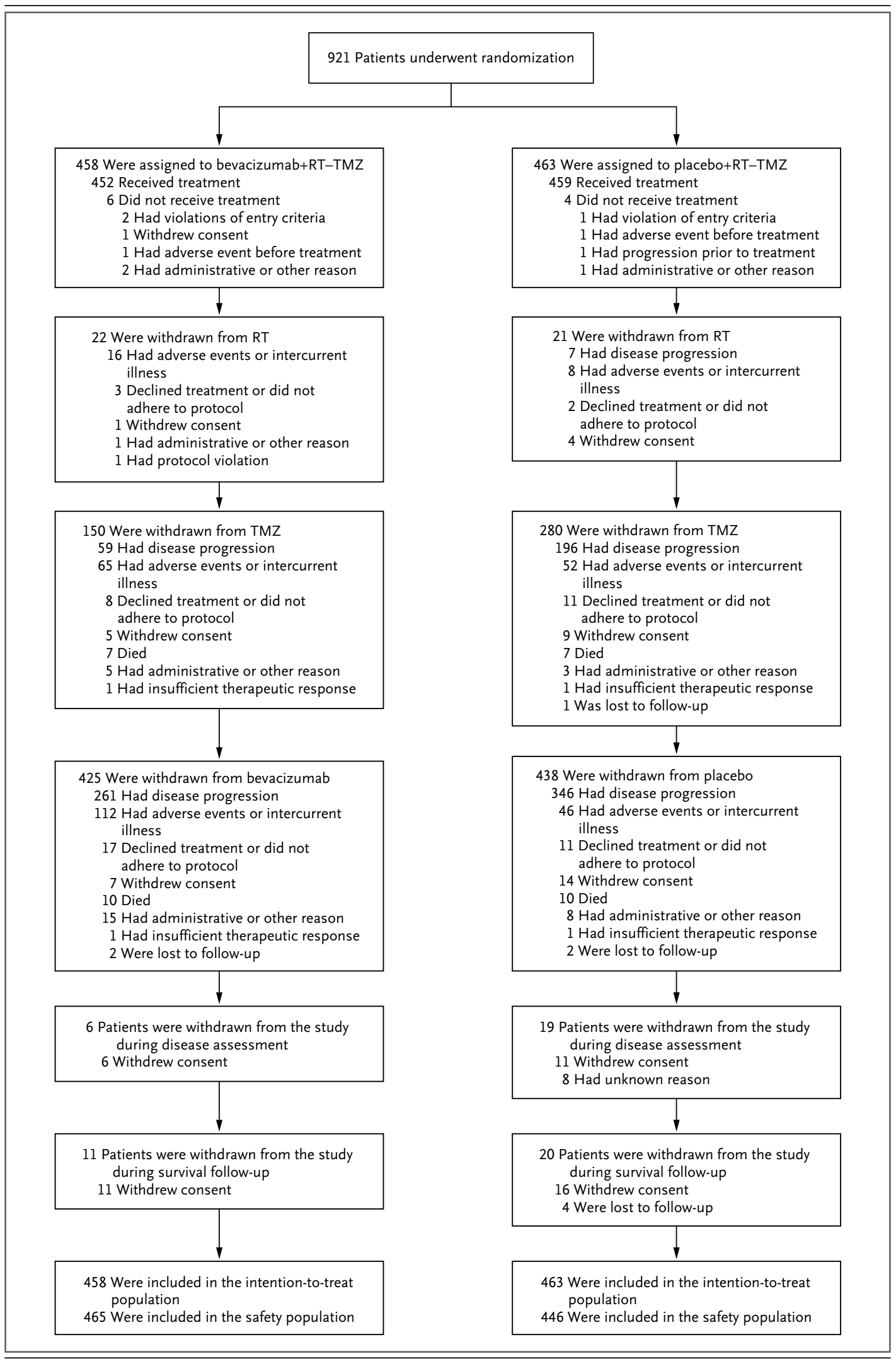

The New England Journal of Medicine

Downloaded from nejm.org at UMEA UNIVERSITY LIBRARY on June 17, 2014. For personal use only. No other uses without permission. Copyright @ 2014 Massachusetts Medical Society. All rights reserved. 
Figure 1 (facing page). Randomization, Treatment, and Follow-up.

Among the 463 patients in the intention-to-treat population who were randomly assigned to placebo, $13 \mathrm{pa}$ tients received at least one dose of bevacizumab and were included in the bevacizumab safety population. RT denotes radiotherapy, and TMZ temozolomide.

(bevacizumab group) and 7.0 months in the group receiving placebo plus radiotherapy-temozolomide (placebo group) (hazard ratio for progression or death with bevacizumab, 0.77), we estimated that 677 events would be required for the study to have $80 \%$ power, with the use of the log-rank test at a two-sided alpha level of $1 \%$. For the analysis of overall survival, assuming a median survival of 18.3 months in the bevacizumab group and 14.6 months in the placebo group (hazard ratio for death, 0.80 ), we estimated that 683 events would be required for the study to have $80 \%$ power, with the use of the log-rank test at a two-sided overall alpha level of $4 \%$.

Two interim analyses were planned for overall survival, and the O'Brien-Fleming group sequential boundary function, in conjunction with the alpha-spending function of Lan and DeMets, was used to adjust for sequential testing of overall survival. ${ }^{31}$ Progression-free survival and overall survival were measured from the date of randomization, and survival estimates were determined with the use of Kaplan-Meier methods. The between-group difference in survival was assessed with the use of a two-sided stratified logrank test. The hazard ratio was estimated with the use of a stratified Cox regression model. Subgroup analyses of progression-free survival and overall survival were prespecified in the statistical analysis plan. Hazard ratios in the subgroups were estimated with the use of an unstratified Cox regression model that included only treatment as a covariate. The planned sample size (920 patients) was based on an assumed enrollment period of 42 months and a follow-up time of at least 17 months for the last patient enrolled, allowing for a $10 \%$ dropout rate for the analysis of progression-free survival at 3 years and a 5\% dropout rate for the analysis of overall survival at 4 years.

Secondary end points included progression-free survival as assessed by independent review, 1-year and 2-year survival rates, safety, and quality of life (as assessed with the use of the QLQ-C30 and BN20). We analyzed quality of life using Kaplan-
Meier methods, applying a specific definition of deterioration-free survival (see Section 2 in the Supplementary Appendix).

Exploratory end points included betweengroup comparisons of glucocorticoid use and Karnofsky performance status. Further details are provided in the Supplementary Appendix.

\section{RESULTS}

\section{PATIENTS}

From June 2009 through March 29, 2011, a total of 921 patients were enrolled at 120 sites in 23 countries; 458 were randomly assigned to the bevacizumab group, and 463 to the placebo group (intention-to-treat population) (Fig. 1). The baseline characteristics of the patients were well balanced between the two groups (Table 1). A total of 911 patients received at least one dose of the study drug (safety population). Patient status as of the clinical cutoff date is shown in Table S2 in the Supplementary Appendix. Most of the patients received the planned treatment doses; the overall durations of treatment and the number of bevacizumab or placebo infusions received were greater in the bevacizumab group than in the placebo group (Table S3 in the Supplementary Appendix). Data-censoring patterns and triggering events at the time of progression of the disease were similar across groups in both the investigator analysis and the independent review (data not shown).

\section{EFFICACY OUTCOMES}

The median progression-free survival was 10.6 months in the bevacizumab group as compared with 6.2 months in the placebo group (stratified hazard ratio for progression or death with bevacizumab, 0.64; 95\% confidence interval [CI], 0.55 to $0.74 ; \mathrm{P}<0.001$ with the use of the log-rank test) (Fig. 2A); these data were confirmed at the time of the survival analysis. The benefit with bevacizumab with respect to progression-free survival was observed across multiple subgroups, including patients with methylated and those with unmethylated MGMT status (Fig. 2B). The independent review also showed significantly longer progression-free survival with bevacizumab than with placebo (median progression-free survival, 8.4 months vs. 4.3 months; stratified hazard ratio for progression or death with bevacizumab, 0.61; $95 \%$ CI, 0.53 to $0.71 ; \mathrm{P}<0.001$ ) (Fig. $\mathrm{S} 1$ in the 
Table 1. Baseline Demographic and Clinical Characteristics.*

\section{Characteristic}

Age - yr

Median

Range

Age - no. (\%)

$<50 \mathrm{yr}$

50-59 yr

60-69 yr

$\geq 70 \mathrm{yr}$

Race or ethnic group — no. (\%)†

White

Black

Asian (Indian subcontinent)

Asian (other than Indian subcontinent)

Native Hawaiian or Pacific Islander

Other

Sex - no. (\%)

Male

Female

Region - no. (\%)

Western Europe

Eastern Europe

Asia

United States

Other

RPA class - no./total no. (\%)

III

IV

$\mathrm{V}$

Karnofsky performance score at baseline - no./total no. (\%) $)$

$$
\text { 50-80 }
$$

$90-100$

MMSE score - no./total no. (\%)』

$<27$

$\geq 27$

WHO performance status - no./total no. (\%) 9

0

1 or 2

MGMT status - \%

Methylated

Nonmethylated

Data missing

Surgical status - no. (\%)

Biopsy only

Partial resection

Complete resection
Bevacizumab plus Radiotherapy and

Temozolomide

$(\mathrm{N}=458)$

Placebo plus

Radiotherapy and

Temozolomide

$(\mathrm{N}=463)$

57

56

20-84

18-79

$116(25.3)$

$158(34.5)$

$145(31.7)$

$39(8.5)$

$413(90.2)$

$3(0.7)$

$4(0.9)$

$35(7.6)$

0

$3(0.7)$

$113(24.4)$

165 (35.6)

$151(32.6)$

$34(7.3)$

419 (90.5)

$4(0.9)$

$2(0.4)$

35 (7.6)

$1(0.2)$

$2(0.4)$

$282(61.6)$

298 (64.4)

$176(38.4)$

$165(35.6)$

$236(51.5)$

77 (16.8)

$34(7.4)$

18 (3.9)

93 (20.3)

$76 / 458$ (16.6)

$261 / 458(57.0)$

$121 / 458$ (26.4)

$149 / 457$ (32.6)

$308 / 457$ (67.4)

$106 / 451$ (23.5)

$345 / 451$ (76.5)

$227 / 458$ (49.6)

$231 / 458(50.4)$

117 (25.5)

225 (49.1)

$116(25.3)$

60 (13.1)

$210(45.9)$

188 (41.0)
237 (51.2)

80 (17.3)

35 (7.6)

18 (3.9)

93 (20.1)

$75 / 462$ (16.2)

279/462 (60.4)

$108 / 462(23.4)$

$140 / 462(30.3)$

$322 / 462$ (69.7)

$108 / 459$ (23.5)

$351 / 459$ (76.5)

238/462 (51.5)

224/462 (48.5)

$120(25.9)$

236 (51.0)

107 (23.1)

44 (9.5)

$223(48.2)$

$196(42.3)$ 
Table 1. (Continued.)

\begin{tabular}{|c|c|c|}
\hline Characteristic & $\begin{array}{l}\text { Bevacizumab plus } \\
\text { Radiotherapy and } \\
\text { Temozolomide } \\
(\mathrm{N}=458)\end{array}$ & $\begin{array}{l}\text { Placebo plus } \\
\text { Radiotherapy and } \\
\text { Temozolomide } \\
(\mathrm{N}=463)\end{array}$ \\
\hline \multicolumn{3}{|l|}{ Histologically confirmed glioblastoma - no. (\%) } \\
\hline Confirmed & $435(95.0)$ & $440(95.0)$ \\
\hline Not confirmed & $9(2.0)$ & $13(2.8)$ \\
\hline Data missing & $14(3.1)$ & $10(2.2)$ \\
\hline \multicolumn{3}{|l|}{ Primary or secondary glioblastoma — no. (\%) } \\
\hline Primary & $452(98.7)$ & $461(100)$ \\
\hline Secondary & $6(1.3)$ & $2(0.4)$ \\
\hline \multicolumn{3}{|l|}{ Quality of life } \\
\hline \multicolumn{3}{|l|}{ Global health status $\|$} \\
\hline No. of patients with completed assessment & 445 & 450 \\
\hline Score & $64.6 \pm 22.4$ & $67.4 \pm 21.0$ \\
\hline \multicolumn{3}{|l|}{ Physical functioning $\|$} \\
\hline No. of patients with completed assessment & 449 & 451 \\
\hline Score & $82.9 \pm 20.1$ & $81.4 \pm 22.4$ \\
\hline \multicolumn{3}{|l|}{ Social functioning $\|$} \\
\hline No. of patients with completed assessment & 448 & 449 \\
\hline Score & $71.7 \pm 29.0$ & $71.6 \pm 28.6$ \\
\hline \multicolumn{3}{|l|}{ Motor functioning $* *$} \\
\hline No. of patients with completed assessment & 449 & 447 \\
\hline Score & $16.8 \pm 23.2$ & $14.8 \pm 20.8$ \\
\hline \multicolumn{3}{|l|}{ Communication deficit*** } \\
\hline No. of patients with completed assessment & 449 & 447 \\
\hline Score & $16.9 \pm 24.8$ & $17.6 \pm 25.2$ \\
\hline \multicolumn{3}{|l|}{ Use of EIAEDs at baseline - no. (\%) } \\
\hline Yes & $87(19.0)$ & $92(19.9)$ \\
\hline No & $371(81.0)$ & $371(80.1)$ \\
\hline \multicolumn{3}{|l|}{ Use of glucocorticoids at baseline - no. (\%) } \\
\hline Yes & $187(40.8)$ & $208(44.9)$ \\
\hline No & $269(58.7)$ & $253(54.6)$ \\
\hline Data missing & $2(0.4)$ & $2(0.4)$ \\
\hline \multicolumn{3}{|c|}{ Time between surgery and first dose of study drug — no./total no. (\%) } \\
\hline$<4 \mathrm{wk}$ & $3 / 452(0.7)$ & $2 / 459(0.4)$ \\
\hline $4-7 w k$ & $435 / 452(96.2)$ & $438 / 459(95.4)$ \\
\hline$>7 \mathrm{wk}$ & $14 / 452(3.1)$ & $19 / 459(4.1)$ \\
\hline
\end{tabular}

* Plus-minus values are means \pm SD. There were no significant between-group differences in any of the characteristics listed here. EIAED denotes enzyme-inducing antiepileptic drug, MGMT O-6-methylguanine-DNA methyltransferase, and RPA recursive partitioning analysis.

$\dagger$ Race or ethnic group was self-reported.

The Karnofsky performance score ranges from 0 to 100, with higher numbers indicating better function.

$\int$ Scores on the Mini-Mental State Examination (MMSE) range from 0 to 30, with higher scores indicating better cognitive function.

I The World Health Organization (WHO) performance status is scored on a scale of 0 to 5 , with 0 indicating fully active, 1 unable to carry out heavy physical work, and 2 up and about more than half the day but unable to work.

$\|$ Scores ranged from 0 to 100 , with higher scores indicating better health-related quality of life.

w* Scores ranged from 0 to 100 , with lower scores indicating better health-related quality of life.

The New England Journal of Medicine 


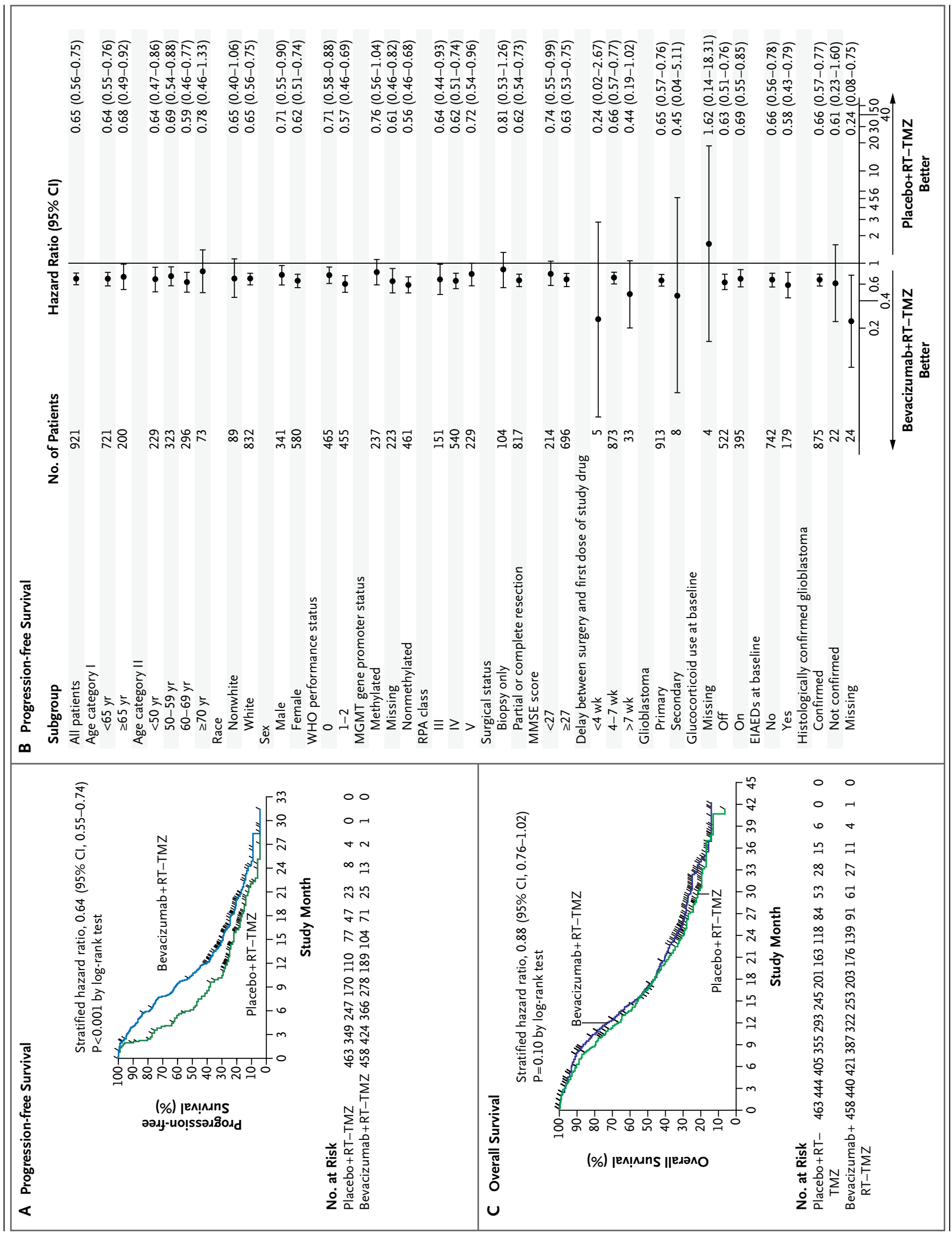


Figure 2 (facing page). Progression-free and Overall Survival.

Panel A shows Kaplan-Meier estimates of investigatorassessed progression-free survival. At the time of this analysis (cutoff date, March 31, 2012), a total of 741 patients ( $80 \%$ of the intention-to-treat population) had had disease progression or had died: 354 patients $(77 \%)$ in the bevacizumab group and 387 patients $(84 \%)$ in the placebo group. Panel B is a forest plot of progressionfree survival according to subgroups (cutoff date for analysis, March 31, 2012). The median follow-up time was 14.4 months in the bevacizumab group and 13.7 months in the placebo group. Panel $\mathrm{C}$ shows Kaplan-Meier estimates of overall survival. At the time of this analysis (cutoff date, February 28, 2013), the median follow-up time was 16.3 months in the bevacizumab group and 15.8 months in the placebo group. Scores on the Mini-Mental State Examination (MMSE) range from 0 to 30 , with higher scores indicating better cognitive function. EIAED denotes enzyme-inducing antiepileptic drug, MGMT O-6-methylguanine-DNA methyltransferase, RPA recursive partitioning analysis, and WHO World Health Organization.

Supplementary Appendix). Pseudoprogression was reported in 10 patients (2.2\%) in the bevacizumab group and in 43 patients $(9.3 \%)$ in the placebo group.

The median overall survival was 16.8 months in the bevacizumab group and 16.7 months in the placebo group (stratified hazard ratio for death with bevacizumab, 0.88 ; $95 \%$ CI, 0.76 to 1.02 ; $\mathrm{P}=0.10$ ) (Fig. 2C). No significant between-group differences in overall survival were observed in the subgroups, including patients with methylated and those with unmethylated MGMT status (Fig. S2 in the Supplementary Appendix). The respective rates of overall survival with bevacizumab and placebo were $72.4 \%$ and $66.3 \%$ at 1 year $(\mathrm{P}=0.049)$ and $33.9 \%$ and $30.1 \%$ at 2 years $(\mathrm{P}=0.24)$.

\section{OTHER CLINICAL MEASURES}

All the patients were required to complete qualityof-life questionnaires. Over 1 year of treatment, the percentage of patients who completed all the quality-of-life questionnaires at an assessment (with assessments performed every 2 months) ranged from 74 to $91 \%$. In the prespecified primary analysis, deterioration-free survival was significantly longer among patients in the bevacizumab group than among those in the placebo group for all five prespecified scales (hazard ratio for deterioration in global health status with bevacizumab, $0.64 ; 95 \% \mathrm{CI}, 0.56$ to $0.74 ; \mathrm{P}<0.001$

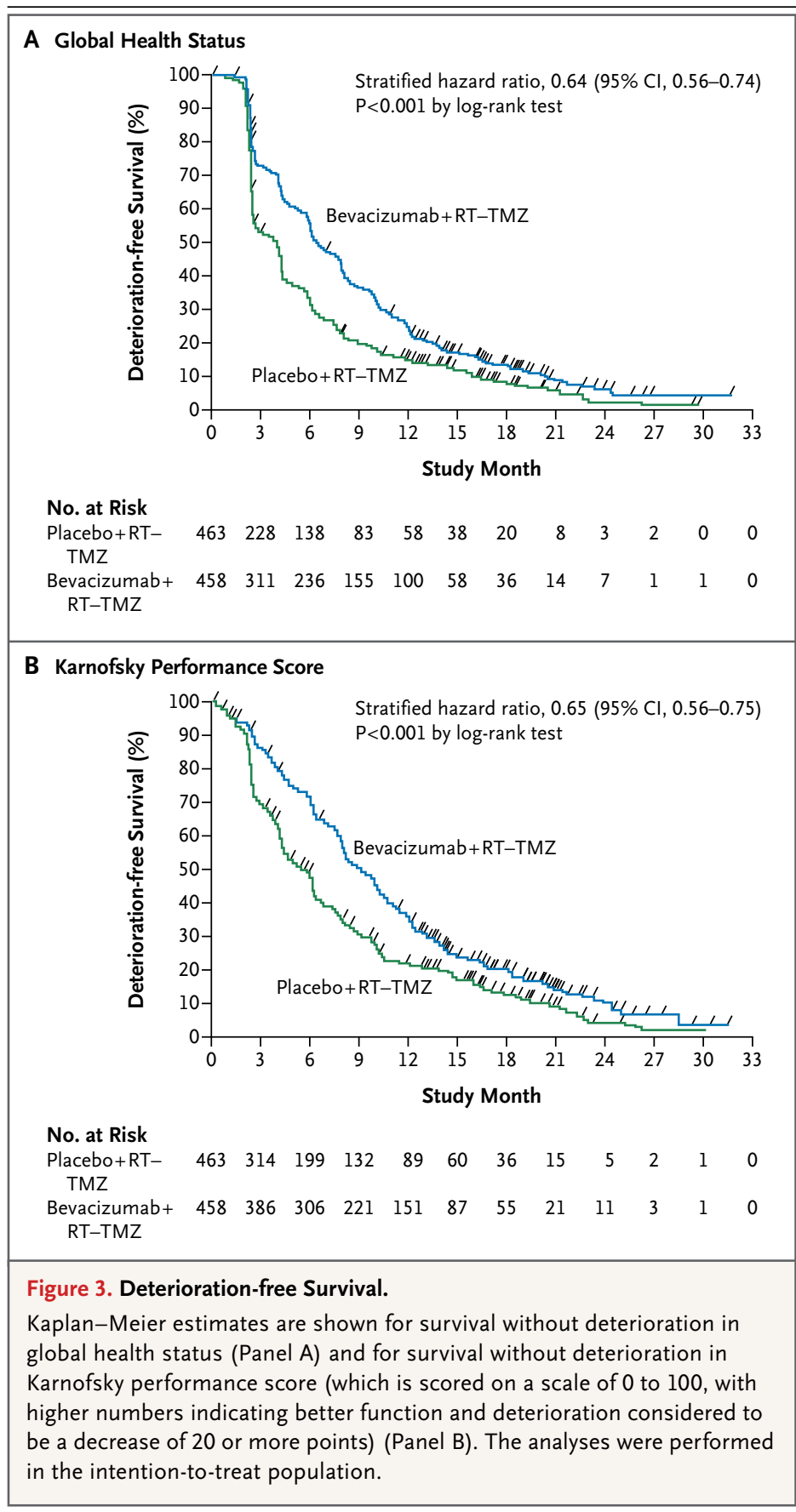

for all comparisons) (Fig. 3A); similarly, in exploratory analyses, deterioration-free survival was significantly longer among patients in the bevacizumab group for all 21 nonprespecified scales ( $\mathrm{P}<0.05$ for all comparisons).

In a sensitivity analysis, the time to deterioration was significantly longer among patients in the bevacizumab group in three of the five pre- 
specified scales (global health status, social functioning, and communication deficit $[\mathrm{P}<0.05$ for these three comparisons]) and in 9 of 21 nonprespecified scales (cognitive functioning, emotional functioning, role functioning, fatigue, visual disorder, weakness in both legs, hair loss, bladder control, and financial difficulties $[\mathrm{P}<0.05$ for all comparisons, not corrected for multiple comparisons]). ${ }^{32}$ The time to deterioration did not differ significantly between the groups for the remaining 2 prespecified scales and 12 nonprespecifed scales. The results of the primary quality-of-life analysis were further validated by exploratory analyses, including analyses of the mean changes in scores from baseline, a mixedmodel-for-repeated-measures analysis, and an analysis of the maintenance of quality of life during progression-free time (data not shown). ${ }^{32}$

The median time that the Karnofsky performance status was maintained at 70 or higher was 9.0 months $(96 \%$ of the progression-free survival time) in the bevacizumab group versus 6.0 months $(97 \%$ of the progression-free survival time) in the placebo group. Survival without deterioration in performance status was significantly longer in the bevacizumab group than in the placebo group (median, 9.0 months vs. 5.5 months; hazard ratio for deterioration with bevacizumab, 0.65 ; $95 \%$ CI, 0.56 to 0.75 ; $\mathrm{P}<0.001$ ) (Fig. 3B); similarly, the time to deterioration in performance status was longer with bevacizumab than with placebo (median, 14.2 months vs. 11.8 months; hazard ratio, $0.79 ; 95 \% \mathrm{CI}, 0.65$ to $0.96 ; \mathrm{P}=0.02$ ).

Most of the patients had normal neurocognitive function at baseline $(76.5 \%$ with an MMSE score $\geq 27$ ) (Table 1). The mean change from baseline in MMSE scores was similar (with overlapping confidence intervals) in the two study groups (Fig. S3 in the Supplementary Appendix). More than $60 \%$ of the patients had a stable or improved MMSE score relative to baseline at each evaluation during progression-free time (Table S4 in the Supplementary Appendix). There was a trend toward a decline in neurocognitive function at the time of progression in both study groups (Fig. S3 in the Supplementary Appendix).

Among patients who were receiving glucocorticoids at baseline (Table 1), glucocorticoid use was discontinued (for $\geq 5$ consecutive days) in $66.3 \%$ of the patients receiving bevacizumab as compared with $47.1 \%$ of the patients receiving placebo. Among patients who were not receiving glucocorticoids at baseline, the time to initiation of glucocorticoids was longer with bevacizumab than with placebo (12.3 months vs. 3.7 months; hazard ratio, $0.71 ; 95 \% \mathrm{CI}, 0.57$ to $0.88 ; \mathrm{P}=0.002$ ) (Fig. S4 in the Supplementary Appendix).

\section{SUBSEQUENT TREATMENT}

A total of 284 patients $(62.0 \%)$ in the bevacizumab group, as compared with 321 (69.3\%) in the placebo group, received some subsequent treatment. Details of salvage treatment are provided in Section 5 in the Supplementary Appendix.

\section{ADVERSE EVENTS}

The median duration of follow-up for safety was 12.3 months in the bevacizumab group and 8.5 months in the placebo group. Adverse events of any grade were reported in $98.5 \%$ of the patients who received bevacizumab and $96.0 \%$ of the patients who received placebo (Table 2). The rate of serious adverse events was higher in the bevacizumab group than in the placebo group (38.8\% vs. $25.6 \%$ ), as were the rates of grade 3 or higher adverse events (66.8\% vs. 51.3\%) and grade 3 or higher adverse events that are often associated with bevacizumab (32.5\% vs. $15.8 \%$ ). The incidences of total and grade 3 or higher arterial thromboembolic events were higher in the bevacizumab group than in the placebo group (Table 2); in 19 of 27 patients with an arterial thromboembolic event in the bevacizumab group (70.4\%) and in 3 of 7 patients with an arterial thromboembolic event in the placebo group (42.9\%), the event resolved. One fatal arterial thromboembolism was documented in each group. Other serious adverse events observed more frequently in the bevacizumab group included bleeding, complications of wound healing, gastrointestinal perforation, and congestive heart failure.

Progressive disease was the most common cause of death in both groups; disease progression was the cause of death in 309 of the 339 patients in the bevacizumab group who died $(91.2 \%)$ and in 301 of the 333 patients in the placebo group who died (90.4\%). Grade 5 adverse events occurred more frequently in the bevacizumab group than in the placebo group (in 20 patients [4.3\%] vs. 12 patients [2.7\%]) (Table 2), as did adverse events leading to discontinuation of treatment (in 122 patients [26.5\%] vs. 61 patients $[13.6 \%])$. 


\section{DISCUSSION}

In this study, bevacizumab combined with standard treatment for patients with newly diagnosed glioblastoma was associated with a 4.4-month increase in median progression-free survival without a significant effect on overall survival. The median investigator-reported progressionfree survival in the placebo group was consistent with that reported previously with the current standard of care for glioblastoma. ${ }^{8}$ The benefit in progression-free survival with bevacizumab according to the independent review was consistent with the benefit according to the investigator assessment. The 2-month difference in progression-free survival between the assessment by the local investigators and the assessment by the independent reviewers may be attributable to more rigid application of imaging criteria by the independent radiologists and the fact that the strict algorithm used in the independent review for determination of progressive disease did not allow for clinical interpretation. Similarly, a difference of 2.8 months between investigator and independent-review assessments was observed in the CENTRIC trial (Cilengitide in Combination with Temozolomide and Radiotherapy in Newly Diagnosed Glioblastoma Phase III Randomized Clinical Trial; ClinicalTrials.gov number, NCT00689221) of cilengitide plus radiotherapy-temozolomide for newly diagnosed glioblastoma. ${ }^{33}$

The adapted Macdonald Response Criteria, which were used to assess progression, anticipated some of the key features of the Response Assessment in Neuro-Oncology (RANO) Working Group criteria, which were not available at the time of the initiation of our study. ${ }^{25,26}$ The adapted Macdonald Response Criteria addressed the observed limitations of imaging assessment by including qualitative evaluation of both noncontrast-enhancing components (by means of $\mathrm{T}_{2}$-weighted imaging or fluid-attenuated inversion recovery [FLAIR]) and small contrastenhancing lesions (at least one diameter $<10 \mathrm{~mm}$ ). These criteria also addressed the transient increases in tumor enhancement (pseudoprogression) associated with front-line chemoradiotherapy by standardizing the assessment of pseudoprogression with the use of a strict algorithm, resulting in lower incidences of pseudoprogression than those in previous studies. ${ }^{34-36}$

No predictive influence of MGMT status or any other subgroup variable was observed with respect to progression-free survival or overall survival. Other prognostic markers for glioblastoma (e.g., surgery status, Karnofsky performance status, age, and recursive partitioning analysis class) were also not predictive of survival, findings that were consistent with those in previous studies. ${ }^{21}$

The addition of bevacizumab to standard radiotherapy-temozolomide therapy as first-line treatment for glioblastoma was also investigated in the phase 3, randomized, placebo-controlled Radiation Therapy Oncology Group (RTOG)-0825 study. That study showed a similar trend toward improvement in progression-free survival (hazard ratio, $0.79 ; 95 \% \mathrm{CI}, 0.66$ to $0.94 ; \mathrm{P}=0.007$ ), with a 3.4-month extension of progression-free survival, although the difference was not significant according to the prespecified alpha level $(\mathrm{P}<0.004)$. No significant difference in overall survival was observed between the study groups (hazard ratio, 1.13; 95\% CI, 0.93 to 1.37; $\mathrm{P}=0.21) .^{37}$

In our study, treatment during progressionfree time was associated with a consistently stable quality of life across all domains, sustained functional independence, and a diminished glucocorticoid requirement. Considering the high baseline quality-of-life scores in this population of patients with newly diagnosed glioblastoma, maintenance of quality of life is a relevant treatment goal. ${ }^{38,39}$ The quality-of-life results of the present study contrast with the results of the RTOG-0825 trial. ${ }^{37,40}$ This may be explained in part by the use of updated imaging criteria in our study that included assessment of nonenhancing tumor and limited the possibility of unrecognized progression that may be associated with a decline in quality of life or neurocognitive function.

A Karnofsky performance status of 70 indicates that the patient is capable of self-care but is not able to perform normal activity or work. The ability to care for oneself is an important treatment goal in glioblastoma and suggests a degree of functional independence. ${ }^{41}$ Patients entered the study with a high performance status and maintained their functional independence (Karnofsky performance status $\geq 70$ ) during treatment and before progression. Furthermore, despite the limitations of the MMSE (e.g., lack of sensitivity), the results of that assessment suggested that neurocognitive function did not decline during progression-free survival in 
either group. Bevacizumab was associated with diminished use of glucocorticoids, which may be a consequence of improved tumor control (reduced tumor mass) as well as the decreased permeability of tumor vasculature afforded by bevacizumab.

The rates of serious adverse events and grade 3 or higher adverse events that are often associated with bevacizumab were higher in the bevacizumab group than in the placebo group, with the difference attributable largely to increased incidences of hypertension and proteinuria. The incidence of arterial thromboembolism was increased in the bevacizumab group as compared with the placebo group, though the majority of episodes resolved, and only one fatal arterial thromboembolism occurred in each group. The safety profile for bevacizumab in this study was consistent with that observed in other clinical settings.

In conclusion, this trial shows that the combination of bevacizumab with standard radiotherapytemozolomide for the treatment of newly diagnosed glioblastoma did not improve overall

\begin{tabular}{|c|c|c|}
\hline \multirow[t]{2}{*}{ Event } & $\begin{array}{l}\text { Bevacizumab plus } \\
\text { Radiotherapy and } \\
\text { Temozolomide } \\
(N=461)\end{array}$ & $\begin{array}{l}\text { Placebo plus } \\
\text { Radiotherapy and } \\
\text { Temozolomide } \\
(\mathrm{N}=450)\end{array}$ \\
\hline & \multicolumn{2}{|c|}{ no. of patients (\%) } \\
\hline Any adverse event & $454(98.5) *$ & $432(96.0)$ \\
\hline Serious adverse event & $179(38.8) *$ & $115(25.6)$ \\
\hline Grade $\geq 3$ adverse event & $308(66.8) *$ & $231(51.3)$ \\
\hline Grade $\geq 3$ adverse event possibly associated with bevacizumab $\uparrow$ & $150(32.5) *$ & $71(15.8)$ \\
\hline Grade 5 adverse event & $20(4.3)$ & $12(2.7)$ \\
\hline Discontinuation of any treatment owing to an adverse event & $122(26.5) *$ & $61(13.6)$ \\
\hline Discontinuation of placebo or bevacizumab owing to an adverse event & $114(24.7) *$ & $46(10.2)$ \\
\hline \multicolumn{3}{|l|}{ Adverse events often associated with bevacizumab $†$} \\
\hline \multicolumn{3}{|l|}{ Bleeding } \\
\hline \multicolumn{3}{|l|}{ Cerebral hemorrhage } \\
\hline All grades & $15(3.3)$ & $9(2.0)$ \\
\hline Grade $\geq 3$ & $9(2.0)$ & $4(0.9)$ \\
\hline \multicolumn{3}{|l|}{ Other, including mucocutaneous bleeding } \\
\hline All grades & $171(37.1) *$ & $88(19.6)$ \\
\hline Grade $\geq 3$ & $6(1.3)$ & $4(0.9)$ \\
\hline \multicolumn{3}{|l|}{ Wound-healing complications } \\
\hline All grades & $32(6.9)$ & $21(4.7)$ \\
\hline Grade $\geq 3$ & $15(3.3)$ & $7(1.6)$ \\
\hline \multicolumn{3}{|l|}{ Arterial thromboembolic events $\mathbb{}$} \\
\hline All grades & $27(5.9) 9$ & $7(1.6)$ \\
\hline Grade $\geq 3$ & $23(5.0) 9$ & $6(1.3)$ \\
\hline \multicolumn{3}{|l|}{ Venous thromboembolic events } \\
\hline All grades & $38(8.2)$ & $43(9.6)$ \\
\hline Grade $\geq 3$ & $35(7.6)$ & $36(8.0)$ \\
\hline \multicolumn{3}{|l|}{ Hypertension } \\
\hline All grades & $181(39.3) *$ & $57(12.7)$ \\
\hline Grade $\geq 3$ & $52(11.3) *$ & $10(2.2)$ \\
\hline \multicolumn{3}{|l|}{ Proteinuria } \\
\hline All grades & $72(15.6) *$ & $19(4.2)$ \\
\hline Grade $\geq 3$ & $25(5.4) *$ & 0 \\
\hline
\end{tabular}




\begin{tabular}{|c|c|c|}
\hline \multicolumn{3}{|l|}{ Table 2. (Continued.) } \\
\hline \multirow[t]{2}{*}{ Event } & $\begin{array}{l}\text { Bevacizumab plus } \\
\text { Radiotherapy and } \\
\text { Temozolomide } \\
(\mathrm{N}=461)\end{array}$ & $\begin{array}{l}\text { Placebo plus } \\
\text { Radiotherapy and } \\
\text { Temozolomide } \\
(\mathrm{N}=450)\end{array}$ \\
\hline & \multicolumn{2}{|c|}{ no. of patients (\%) } \\
\hline \multicolumn{3}{|c|}{ Gastrointestinal perforation, including fistula or abscess } \\
\hline All grades & $8(1.7)$ & $2(0.4)$ \\
\hline Grade $\geq 3$ & $5(1.1)$ & $1(0.2)$ \\
\hline \multicolumn{3}{|l|}{ Abscesses and fistulae } \\
\hline All grades & $2(0.4)$ & $3(0.7)$ \\
\hline Grade $\geq 3$ & $2(0.4)$ & $3(0.7)$ \\
\hline \multicolumn{3}{|l|}{ Congestive heart failure } \\
\hline All grades & $2(0.4)$ & $1(0.2)$ \\
\hline Grade $\geq 3$ & $2(0.4)$ & 0 \\
\hline \multicolumn{3}{|l|}{ Other adverse events } \\
\hline Grade $\geq 3$ thrombocytopenia & $69(15.0) \|$ & $44(9.8)$ \\
\hline Grade $\geq 3$ fatigue & $34(7.4)$ & $21(4.7)$ \\
\hline
\end{tabular}

$* \mathrm{P}<0.001$ for the comparison between groups.

$\dagger$ Adverse events that are often associated with bevacizumab treatment include hypertension; proteinuria; gastrointestinal perforation; complications related to wound healing (including craniotomy); thromboembolic events (venous thrombosis and arterial thrombosis); bleeding, including mucocutaneous bleeding, cerebral hemorrhage, and other hemorrhages; congestive heart failure; abscesses and fistulae; and the posterior reversible encephalopathy syndrome.

$\mp$ Adverse events leading to death in the bevacizumab group were infections (in 8 patients), pulmonary embolism (in 2), respiratory or lung disorders (in 1), cardiac disorders (in 3), general deterioration in physical health (in 2), gastrointestinal perforation (in 1), brain edema (in 1), hepatotoxicity (in 1), and tumor hemorrhage (in 1). Adverse events leading to death in the placebo group were infections (in 6 patients), pulmonary embolism (in 1), cardiorespiratory arrest (in 1), general disorders (in 1), gastrointestinal hemorrhage (in 1), liver metastases (in 1), and cerebrovascular accident (in 1).

$\int A$ total of 21 of the 27 total arterial thromboembolic events (78\%) in the bevacizumab group were serious events and 5 of the 7 total arterial thromboembolic events (71\%) in the placebo group were serious events.

I $\mathrm{P}=0.001$ for the between-group comparison of all grades, and $\mathrm{P}=0.003$ for the comparison of grade 3 or higher.

$\| \mathrm{P}=0.02$ for the comparison between groups.

survival but resulted in a 4.4-month improvement in median progression-free survival, with quality of life and functional status maintained; however, there was an increase in adverse events associated with bevacizumab therapy. The benefit in progression-free survival was observed consistently across subgroups.
Supported by F. Hoffmann-La Roche.

Disclosure forms provided by the authors are available with the full text of this article at NEJM.org.

We thank the patients and their families; the AVAglio study coordinators and nurses; Yannick Kerloeguen, Ph.D., Ana Maria Abajo Guijarro, Ph.D., and Josep Garcia, Ph.D., of F. Hoffmann-La Roche for data analysis and interpretation; and Emma McConnell, Ph.D., and Tracy McNally, Ph.D., of Gardiner-Caldwell Communications for medical writing support (funded by F. Hoffmann-La Roche).
REFERENCES

1. Ohgaki H. Epidemiology of brain tumors. Methods Mol Biol 2009;472:323-42.

2. Central Brain Tumor Registry of the United States (CBTRUS) home page (http:// www.cbtrus.org/2011-NPCR-SEER/ WEB-0407-Report-3-3-2011.pdf).

3. Bosma I, Vos MJ, Heimans JJ, et al. The course of neurocognitive functioning in high-grade glioma patients. Neuro Oncol 2007;9:53-62

4. Meyers CA, Hess KR. Multifaceted end points in brain tumor clinical trials: cognitive deterioration precedes MRI progression. Neuro Oncol 2003;5:89-95.

5. Giovagnoli AR, Silvani A, Colombo E, Boiardi A. Facets and determinants of quality of life in patients with recurrent high grade glioma. J Neurol Neurosurg Psychiatry 2005;76:562-8.

6. Henriksson R, Asklund T, Poulsen HS. Impact of therapy on quality of life, neurocognitive function and their correlates in glioblastoma multiforme: a review. J Neurooncol 2011;104:639-46.
7. Klein M, Taphoorn MJ, Heimans JJ, et al. Neurobehavioral status and healthrelated quality of life in newly diagnosed high-grade glioma patients. J Clin Oncol 2001;19:4037-47. [Erratum, J Clin Oncol 2003;21:2628.]

8. Stupp R, Mason WP, van den Bent MJ, et al. Radiotherapy plus concomitant and adjuvant temozolomide for glioblastoma. N Engl J Med 2005;352:987-96.

9. National Comprehensive Cancer Network. NCCN guidelines: central nervous 
system cancers. Version 1. 2012 (http:/l www.nccn.org/professionals/physician_gls/ pdf/cns.pdf).

10. Stupp R, Tonn J-C, Brada M, Pentheroudakis G. High-grade malignant glioma: ESMO Clinical Practice Guidelines for diagnosis, treatment and follow-up. Ann Oncol 2010;21:Suppl 5:v190-v193.

11. Macdonald DR, Kiebert G, Prados M, Yung A, Olson J. Benefit of temozolomide compared to procarbazine in treatment of glioblastoma multiforme at first relapse: effect on neurological functioning, performance status, and health related quality of life. Cancer Invest 2005;23:138-44.

12. Plate $\mathrm{KH}$, Breier G, Weich HA, Risau W. Vascular endothelial growth factor is a potential tumour angiogenesis factor in human gliomas in vivo. Nature 1992;359: 845-8.

13. Berkman RA, Merrill MJ, Reinhold WC, et al. Expression of the vascular permeability factor/vascular endothelial growth factor gene in central nervous system neoplasms. J Clin Invest 1993;91:153-9.

14. Hicklin DJ, Ellis LM. Role of the vascular endothelial growth factor pathway in tumor growth and angiogenesis. J Clin Oncol 2005;23:1011-27.

15. Chinot OL, de La Motte Rouge T, Moore $\mathrm{N}$, et al. AVAglio: Phase 3 trial of bevacizumab plus temozolomide and radiotherapy in newly diagnosed glioblastoma multiforme. Adv Ther 2011;28: 334-40.

16. Chi AS, Sorensen AG, Jain RK, Batchelor TT. Angiogenesis as a therapeutic target in malignant gliomas. Oncologist 2009;14:621-36.

17. Vredenburgh JJ, Desjardins A, Herndon JE II, et al. Bevacizumab plus irinotecan in recurrent glioblastoma multiforme. J Clin Oncol 2007;25:4722-9.

18. Friedman HS, Prados MD, Wen PY, et al. Bevacizumab alone and in combination with irinotecan in recurrent glioblastoma. J Clin Oncol 2009;27:4733-40.

19. Kreisl TN, Kim L, Moore K, et al. Phase II trial of single-agent bevacizumab followed by bevacizumab plus irinotecan at tumor progression in recurrent glioblastoma. J Clin Oncol 2009;27:740-5.

20. Gutin PH, Iwamoto FM, Beal K, et al. Safety and efficacy of bevacizumab with hypofractionated stereotactic irradiation for recurrent malignant gliomas. Int J Radiat Oncol Biol Phys 2009;75:156-63.

21. Lai A, Tran A, Nghiemphu PL, et al. Phase II study of bevacizumab plus temozolomide during and after radiation therapy for patients with newly diagnosed glioblastoma multiforme. J Clin Oncol 2011;29:142-8.

22. Narayana A, Gruber D, Kunnakkat $S$, et al. A clinical trial of bevacizumab, temozolomide, and radiation for newly diagnosed glioblastoma. J Neurosurg 2012; 116:341-5.

23. Mirimanoff RO, Gorlia T, Mason W, et al. Radiotherapy and temozolomide for newly diagnosed glioblastoma: recursive partitioning analysis of the EORTC 26981/22981-NCIC CE3 phase III randomized trial. J Clin Oncol 2006;24:2563-9.

24. Temodal, SmPC (product information). Surrey, United Kingdom: electronic Medicines Compendium (eMC) (http:// www.medicines.org.uk/emc/document .aspx?documentid=7027).

25. Chinot OL, Macdonald DR, Abrey LE, Zahlmann G, Kerloëguen Y, Cloughesy TF. Response assessment criteria for glioblastoma: practical adaptation and implementation in clinical trials of antiangiogenic therapy. Curr Neurol Neurosci Rep 2013;13:347.

26. Wen PY, Macdonald DR, Reardon DA, et al. Updated response assessment criteria for high-grade gliomas. J Clin Oncol 2010;28:1963-72.

27. Aaronson NK, Ahmedzai S, Bergman $\mathrm{B}$, et al. The European Organization for Research and Treatment of Cancer QLQ-C30 a quality-of-life instrument for use in international clinical trials in oncology. J Natl Cancer Inst 1993;85:365-76.

28. Osoba D, Aaronson NK, Muller M, et al. The development and psychometric validation of a brain cancer quality-oflife questionnaire for use in combination with general cancer-specific questionnaires. Qual Life Res 1996;5:139-50.

29. Taphoorn MJ, Claassens L, Aaronson $\mathrm{NK}$, et al. An international validation study of the EORTC brain cancer module (EORTC QLQ-BN20) for assessing healthrelated quality of life and symptoms in brain cancer patients. Eur J Cancer 2010; 46:1033-40.

30. Common Terminology Criteria for Adverse Events v3.0 (CTCAE). Bethesda, MD Cancer Therapy Evaluation Program, 2006 (http://ctep.cancer.gov/protocolDevelopment/ electronic_applications/docs/ctcaev3.pdf). 31. Lan KKG, DeMets DL. Discrete sequential boundaries for clinical trials. Biometrika 1983;70:659-63.

32. Henriksson R, Bottomley A, Mason $\mathrm{W}$, et al. Progression-free survival (PFS) and health-related quality of life (HRQoL) in AVAglio, a phase III study of bevacizumab (Bv), temozolomide (T), and radio- therapy (RT) in newly diagnosed glioblastoma (GBM). Presented at the 2013 Annual Meeting of the American Society of Clinical Oncology, Chicago, May 31June 4, 2013. abstract.

33. Stupp R, Hegi ME, Gorlia T, et al. Cilengitide combined with standard treatment for patients with newly diagnosed glioblastoma and methylated O6-methylguanine-DNA methyltransferase (MGMT) gene promoter: key results of the multicenter, randomized, open-label, controlled, phase III CENTRIC study. Presented at the 2013 Annual Meeting of the American Society of Clinical Oncology, Chicago, May 31-June 4, 2013. abstract.

34. Brandes AA, Tosoni A, Spagnolli F, et al. Disease progression or pseudoprogression after concomitant radiochemotherapy treatment: pitfalls in neurooncology. Neuro Oncol 2008;10:361-7.

35. Brandsma D, Stalpers L, Taal W, Sminia P, van den Bent MJ. Clinical features, mechanisms, and management of pseudoprogression in malignant gliomas. Lancet Oncol 2008;9:453-61.

36. Brandes AA, Franceschi E, Tosoni A, et al. MGMT promoter methylation status can predict the incidence and outcome of pseudoprogression after concomitant radiochemotherapy in newly diagnosed glioblastoma patients. J Clin Oncol 2008; 26:2192-7.

37. Gilbert MR, Dignam JJ, Armstrong TS, et al. A randomized trial of bevacizumab for newly diagnosed glioblastoma. N Engl J Med 2014;370:699-708.

38. Osoba D, Brada M, Yung WK, Prados M. Health-related quality of life in patients treated with temozolomide versus procarbazine for recurrent glioblastoma multiforme. J Clin Oncol 2000;18:1481-91. 39. Brada M, Hoang-Xuan K, Rampling R, et al. Multicenter phase II trial of temozolomide in patients with glioblastoma multiforme at first relapse. Ann Oncol 2001;12:259-66.

40. Armstrong TS, Won M, Wefel JS, et al. Comparative impact of treatment on patient reported outcomes (PROs) in patients with glioblastoma (GBM) enrolled in RTOG 0825. Presented at the 2013 Annual Meeting of the American Society of Clinical Oncology, Chicago, May 31-June 4, 2013. abstract.

41. Tabouret E, Barrie M, Thiebaut A, et al. Limited impact of prognostic factors in patients with recurrent glioblastoma multiforme treated with a bevacizumab-based regimen. J Neurooncol 2013;114:191-8. Copyright (c) 2014 Massachusetts Medical Society.

POSTING PRESENTATIONS FROM MEDICAL MEETINGS ONLINE Online posting of an audio or video recording of an oral presentation at a medical meeting, with selected slides from the presentation, is not considered prior publication. Authors should feel free to call or send e-mail to the Journal's Editorial Offices if there are any questions about this policy. 